\title{
Functionalized nanopipettes: toward label-free, single cell biosensors
}

\author{
Paolo Actis • Andy C. Mak • Nader Pourmand
}

Received: 15 February 2010 / Accepted: 26 May 2010 /Published online: 19 June 2010

(C) The Author(s) 2010. This article is published with open access at Springerlink.com

\begin{abstract}
Nanopipette technology has been proven to be a label-free biosensor capable of identifying DNA and proteins. The nanopipette can include specific recognition elements for analyte discrimination based on size, shape, and charge density. The fully electrical read-out and the ease and low-cost fabrication are unique features that give this technology an enormous potential. Unlike other biosensing platforms, nanopipettes can be precisely manipulated with submicron accuracy and used to study single cell dynamics. This review is focused on creative applications of nanopipette technology for biosensing. We highlight the potential of this technology with a particular attention to integration of this biosensor with single cell manipulation platforms.
\end{abstract}

Keywords Nanopipette - Label-free - Single cell detection . Single molecule detection $\cdot$ Biosensor $\cdot$ Nanopore

\section{Introduction}

Increasing demand for more sensitive analytical and diagnostic tools for the identification of biomolecules has benefited from advanced nanofabrication techniques and the significant strides made in the biosensing and biomed-

P. Actis · A. C. Mak $\cdot$ N. Pourmand $(\bowtie)$

Department of Biomolecular Engineering,

University of California,

Santa Cruz, 1156 High Street,

Santa Cruz, CA 95064, USA

e-mail: pourmand@soe.ucsc.edu

\section{A. C. Mak}

Stanford Genome Technology Center, Stanford University,

855 California Ave,

Palo Alto, CA 94304, USA ical research fields in recent years. New biosensing techniques such as nanostructured optical fiber arrays [1], carbon nanotube biosensors [2], nanowires [3], and nanoparticles [4] provide improved sensitivity and often require less complicated instrumentation than existing molecular detection technology. A relatively new label-free technology, the nanopipette illustrates the trend toward exciting new approaches for biosensing applications.

A pipette can be loosely defined as a hollow structure in which the cavity acts as a passage for the dispensation of fluid from one region to the next. Typically, pipettes are categorized by their volume-microliter, nanoliter, pico, and zeptoliter pipettes [5]. The volumetric definition generally provides a good guideline on the pipette dimensions. However, when the pipette is used as a biosensor (rather than to dispense a liquid volume), the tip diameter is of greater interest than the overall volume. One can conceivably apply a similar SI standard (micro-, nano-, etc.) to the tip diameter. But a more descriptive subdivision is more desirable and often more informative.

Although the SI definitions of the prefixes are clear (micro-, nano-, etc.), the modifiers to the prefixes are often not bound by the same set of standards. For instance, electrochemists had previously struggled with the nomenclature of electrodes based on their size. It became necessary to have some standardization for the description of the electrodes. Electrodes with a diameter of $<200 \mathrm{~nm}$ are defined as nanoelectrodes, whereas microelectrodes are normally considered to be $\sim 0.2$ $20 \mu \mathrm{m}$ in diameter, according to the IUPAC classification $[6,7]$. Applying similar standardization, one can define nanopipettes to be hollow, free standing structures with an opening in the 1-200 $\mathrm{nm}$ range. Although a definition based on simple geometrical parameter is exhaustive, nanopipettes with openings smaller than $\sim 100 \mathrm{~nm}$ show 
non-uniform electrochemical characteristics stemming from the physical properties at the nanoscale, leading to new physical behavior, as described below in the "Electrochemistry of nanopipettes" section.

While nanopipettes and solid-state nanopores are fundamentally similar in terms of the sensing principle, the fabrication processes and the configuration of the components suggest that the two technologies should be considered two different entities. Here we explore and review the fundamental elements of nanopipettes and recent works from various groups related to the creative use of nanopipettes as biosensors. The applications of nanopipettes to investigate single molecule biophysics and to image cells at the nanoscale $[8,9]$ have been recently reviewed and will not be discussed here.

Nanopipettes offer a unique biosensing platform that is potentially capable of detecting single molecules near their most sensitive region. Typically an elongated cone, the dimension and geometry of the nanopipette tip orifice are crucial for the development of a new sensitive biosensing platform. The nanopipette has a size comparable to that of DNA and protein. The interaction of such a biomolecules with the nanopipette pore causes two unique eventstemporary blockage due to ionic/molecular translocation through the nanopipette and/or permanent blockage resulting from binding to a tip that has been functionalized with specific recognition molecules. Each of these interactions causes a distinctive change of the nanopipette electrical behavior. The electrical changes can then be detected with simple electrochemistry without the need for labeling the molecule of interest. The detection principles are detailed in the latter part of this review.

Our group, along with others, has reported the use of different materials for nanopipette fabrication. The diversity of prospective materials, glass or metallic, makes nanopipettes extremely versatile systems toward biosensor development. Each scheme can be tailored with a specific material for optimal sensitivity and robustness. While routinely used as electrochemical sensors $[10,11]$, nanopipettes can readily couple to other instrumentation for secondary detection [12] or parallel verification [13]. The potential combination with other analytical techniques has led to a multitude of chemical and biochemical detection approaches [14-16]. In this review, we will focus on the recent novel, label-free, biosensing applications using nanopipette. Also, we will explore the potential of similar structures to be used as biosensors and diagnostic tools.

\section{Nanopipette materials and fabrication}

Interest in nanopipettes was triggered by significant advances in nanofabrication techniques [17]. A wide range of nano- pipettes have been fabricated under different names with various materials for chemical and biochemical sensing. One of the most attractive features of nanopipettes is the simplicity of the fabrication. Due to their inherent stability and unique thermal properties, glass substrates have been a leading choice for nanopipette fabrication. The low melting temperature of borosilicate compared to fused quartz makes the fabrication of sub-100 nm nanopipettes is extremely difficult [18]. Besides this technical limitation, quartz offers superior material properties for a variety of research applications. For example, quartz is stronger than other glasses and can facilitate penetration through tough tissues which would normally break borosilicate pipettes [19]. In electrochemical applications, quartz has the lowest electrical noise among all types of glass available [20, 21]. Furthermore, quartz contains none of the metals used in conventional glasses, making it virtually free from fluorescence when illuminated [22]. As a result, fused quartz is the predominant glass substrate for fabricating nanopipettes [23].

The most widely adopted fabrication scheme employs laser pulling of a glass capillary, which is easy and costeffective. However, fabrication of nanopipettes with nanometer precision is technically challenging. Gao and colleagues [24] developed an etching protocol to enhance the reproducibility of the pore size. First the capillary was pulled into a micropipette and then the tip was melted to completely close the terminal. A nanopore was opened at the very tip by external etching and monitored through electrochemical measurements, in order to attain the desired pore size (Fig. 1).

Significant effort has been invested in fabricating nanopipettes from materials other than glass. Kim and colleagues [25] fabricated carbon-based nanopipettes with large aspect ratios (length/diameter) based on the glasspulling technique. In this technique, carbon layers are deposited onto the exterior and interior of the pulled aluminosilicate nanopipettes using catalytic chemical vapor deposition. The exterior carbon layer and the glass layer are subsequently removed by chemical etching, exposing the interior carbon nanopipette tip structure.

Freedman and coworkers [26] fabricated carbon nanotube-tipped probe using magnetic techniques. In this approach, a magnetized carbon nanotube (mCNT) is affixed to the tip of a conventional glass nanopipette using magnetic manipulation. The resulting mCNT-tipped nanopipettes were sufficiently robust that they could be used to penetrate cell membranes. The nanopipette also demonstrated fluidic transport ability through the opening of the mCNTs.

In general, nanopipettes can be fabricated with many different materials, each providing unique characteristics and electrochemical properties. The one significant advantage of adopting nanopipettes is that the fabrication 
Fig. 1 Schematic representation of the etching process of a sealed nanopipette. a Microscopic image of the sealed micropipette tip. b, $\mathbf{c}$ The external etching was monitored via electrochemical measurements until the desired pore size was obtained. Adapted with permission from [20]


process is extremely simple, or even considered fab-less, in some cases.

\section{Electrochemistry of nanopipettes}

The electrochemical behavior of nanopipettes deviates significantly from that of conventional microelectrodes. For instance, glass nanopipettes respond to a symmetric voltage input by exhibiting an asymmetric output current, an effect recognized as current rectification [27]. This phenomenon is attributed to the formation of a diffuse electrical double layer $(d d l)$ within the tip orifice. When the $d d l$ thickness is comparable to the diameter of the nanopipette, the electrostatic interaction between ionic species and surface charges will affect ion transport properties. Figure 2 demonstrates current rectification for nanopipettes with different surface charges.

Current rectification has been shown to be affected by electrolyte concentration, $\mathrm{pH}$, and applied voltage [28]. Current rectification is only observed at electrolyte concentrations at or below $100 \mathrm{mM}$. No significant current rectification was reported at high electrolyte concentrations. Consistent with existing electrochemical literature, the $d d l$ thickness is reduced with an increase of electrolyte concentration, e.g., characteristic $d d l$ thicknesses are about 3 , 1 , and $0.3 \mathrm{~nm}$ in $0.01,0.1$, and $1 \mathrm{M} \mathrm{KCl}$ solutions, respectively [29]. At high salt concentration, the ion flow becomes insensible to surface charges. Therefore the overall electrolyte concentration in the system is crucial for nanopipette experiments.

Current rectification can be modulated by functional layers deposited or covalently attached to the nanopipette opening region. Poly-1-lysine, a polypeptide bearing positively charged amino groups, can be physisorbed on the negatively charged nanopipette surface [28]. The protonated amino groups invert the current rectification (Fig. 2c). Similar results were observed with nanopipette modified with cationic dendrimers [30] and in a protein-binding study using PEG-modified nanopipette-like structures [31].
The degree of rectification, or rectification coefficient $(r)$, is defined as the ratio of absolute values of current recorded at a given negative and the same positive voltage,

$r=\left|\frac{I_{-}}{I_{+}}\right|$

The rectification coefficient is a useful parameter for monitoring the variation of nanopipette electrical response with the introduction of a functionalized layer to the nanopipette tips. Uncoated glass nanopipette surfaces (e.g., quartz or borosilicate) induce a negative current rectification $(r>1)$. Functionalization of the nanopipette quartz surface with positively charged polyelectrolytes, such as poly-l-lysine, inverts the current rectification $(r<1)$. In general, any charged molecule captured at the nanopipette tip will modify the surface charge density at the nanopipette and the binding can be monitored by plotting the variation of the rectification coefficient over time. Such data provide details of binding or adsorption events that occur at the tip orifice.

\section{Modeling}

To better understand how nanopipettes can be employed as biosensors, it is important to understand how the nanopipette resistance is determined, as the sensed signal with a nanopipette mainly reflects changes of its total resistance. The nanopipette resistance can be described by the equation:

$R=\frac{\rho l}{\pi r_{s}^{2}}+\frac{\rho \cot (\theta / 2)}{\pi}\left(\frac{1}{r_{t}}-\frac{1}{r_{s}}\right)$

Where $\rho$ is resistivity, $l$ is the length of the shank; $r_{s}$ and $r_{t}$ are the radii of the shank and the tip opening (Fig. 2a), respectively, and $\theta$ is the cone angle [32].

Typically, the radius of the shank is far greater than that of the tip opening (i.e., $r_{s}>>r_{t}$ ). Therefore the total resistance, $R$, is dominated by the tip radius of the nanopipette in the second part of the equation. Resistance of the nanopipette decreases as the distance from the orifice 
Fig. 2 a Geometrical representation of a nanopipette tip. $\mathbf{b}$ Bare and $\mathbf{c}$ PLL-coated electrical double layer formed at the inner surface of nanopipette electrode in solution and associated current/voltage plots

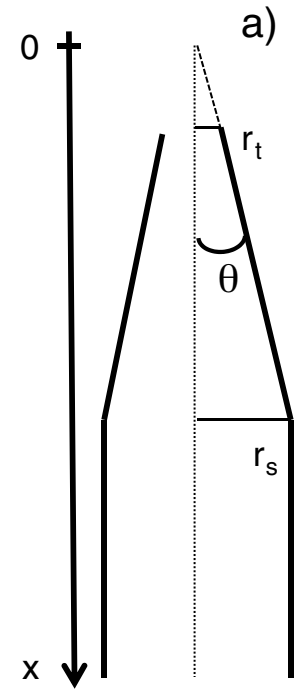

increases. If one replaces $r_{s}$ with $r_{x}(x)$ in Eq. 1, the resistance becomes expressed as the partial resistance as a function of $x$ (the defined $x$-axis is identical to the nanopipette symmetry axis, with its origin at the extrapolated apex of the cone):

$R(x)=\frac{\rho \cot (\theta / 2)}{\pi}\left(\frac{1}{r_{t}}-\frac{1}{r_{x}(x)}\right)$

and $r_{x}(x)=x \tan (\theta / 2)$. Equation 2 demonstrates that, the larger $r_{x}(x)$ gets, the less significant the resistance becomes, thus the majority of the resistance is focused at the proximity of the tip and then the Eq. 2 can be reduced to:

$R \cong \frac{\rho \cot (\theta / 2)}{\pi r_{t}}$

Equation 3 gives an approximate relationship between the nanopipette orifice size and by simple resistance measurements and it is in good agreement with radii estimated by the electron microscope [23, 33].

It is important to point out that the confinement of the resistance at the very tip of a nanopipette makes this technology extremely appealing for the development of ultrasensitive biomolecular detection platforms capable of detecting single molecule events [8, 34].

\section{Experimental considerations}

Since the current flowing through the nanopipette is too small to polarize a reference electrode [27], a two-electrode setup is generally used in nanopipette experiments. A typical setup is shown in Fig. 3. The nanopipette, acting as the working electrode, is backfilled with an electrolyte solution, and a $\mathrm{Ag} / \mathrm{AgCl}$ electrode is inserted. Another $\mathrm{Ag} /$
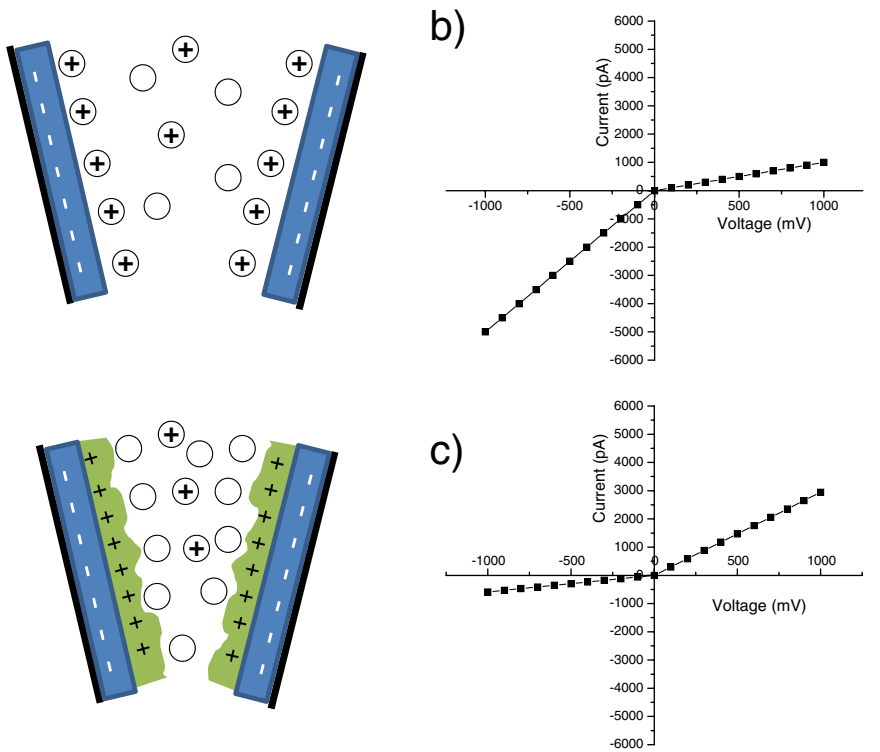

$\mathrm{AgCl}$ electrode is placed in bulk solution to act as an auxiliary/reference electrode. Both electrodes are connected to amplifiers and the current-voltage measurements can be computer-controlled. Since nanobubbles are the dominant source of electrical noise in solid-state nanopores [35], every solution needs to be degassed prior to use. The electrical noise characteristics of ionic current can be further reduced by treating the glass capillaries with sulfuric acid/hydrogen peroxide ("piranha solution") before laser pulling [36].

Generally, the input voltage is crucial to the sensing and detection mechanism. Symmetric waveforms, such as sine waves or linear sweep, are useful for investigating the binding of charged molecules (such as DNA) and binding kinetics can be monitored by following the variation of the rectification coefficient over time. On the other hand, a constant voltage will not allow any monitoring of the change in the rectification properties but it is more suitable

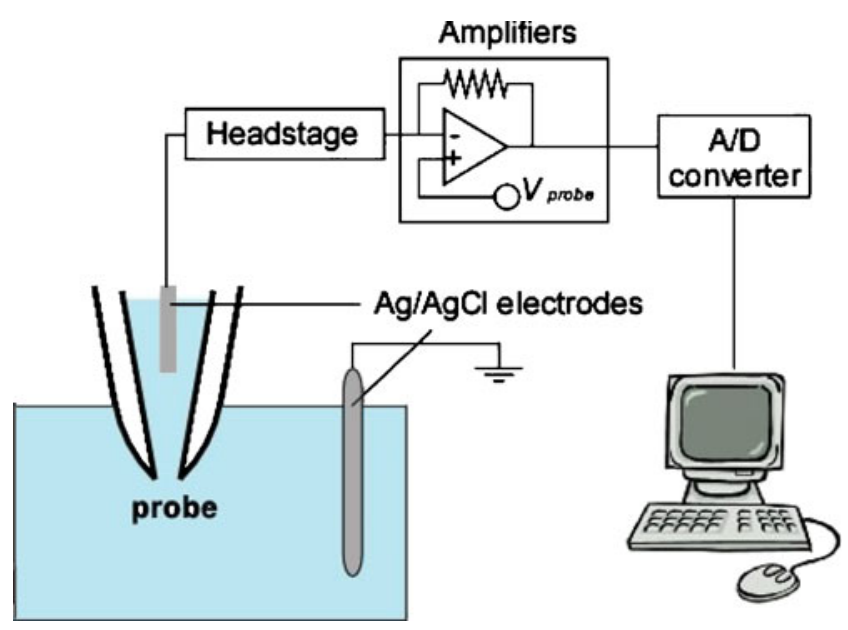

Fig. 3 Typical nanopipette detection platform 
for discriminating binding events of neutral or slightly charged molecules. In addition, a constant voltage can concentrate molecules at the tip though diffusion, electromigration, and electro-osmotic flow [10].

\section{Nanopipettes as electrochemical biosensors}

Nanopipettes have been used for the detection of ionic current blockade events caused by the translocation of DNA labeled with a gold nanoparticles through the $50 \mathrm{~nm}$ nanopipette pore [23] (Fig. 4). The nanopipette assay can uniquely discriminate nanoparticle (head) from oligo (tail) events.

A systematic statistical analysis showed a drastic difference between blockades generated by the oligo (tail) and the nanoparticle (head). The frequency of oligo-tail events was three times higher compared to particle head with mean blockade intensity of about $10 \mathrm{pA}$, as opposed $25 \mathrm{pA}$ for particle head blockades (Fig. 5).

The process of trapping and translocation of these conjugates through the nanopipette is not the result of a simple diffusion mechanism with Gaussian distribution as it would be for Brownian motion, but a mechanism by which molecules must cross an energy barrier in order to enter or escape across the pore. This first attempt to employ a nanopipette as a biosensor clearly showed some potential, and an appropriate surface chemistry will enhance this approach, turning it into a label-free assay for DNA without the use of nanoparticles.

Starting with these considerations, $\mathrm{Fu}$ and colleagues [30] functionalized the nanopipette surface with dendrimers to detect hybridization of specific DNA strands. A particular class of dendrimer, G4-PAMAM, creates a highly cationic surface of known charge that was employed for the electrostatic binding of probe DNA strands. Currentvoltage measurements were performed using linear sweep voltammetry, and the rectification coefficient was interpreted to reflect the DNA binding event. Probe DNA ( $5 \mu \mathrm{M}$ solution) was electrostatically adsorbed onto the nanopipette surface. The initial assessment of the surface using the rectification coefficient indicated that all surface adsorption sites were saturated. Addition of complementary

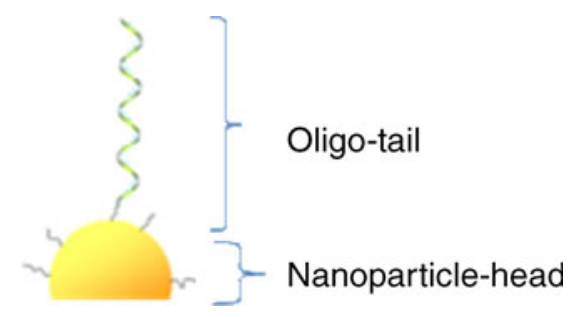

Fig. 4 Schematic representation of a 10-nm gold nanoparticle-labeled oligo. Not to scale

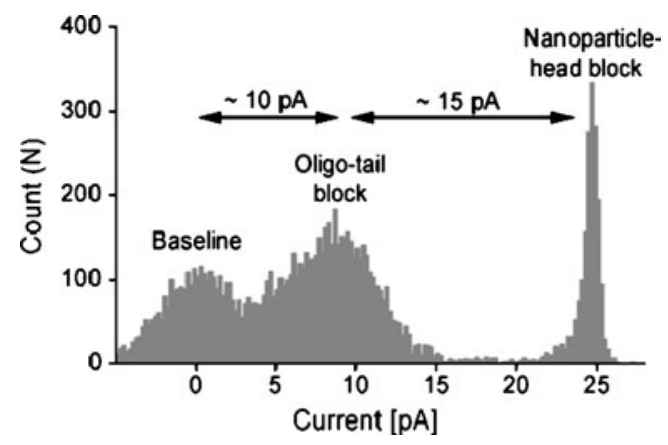

Fig. 5 Statistical distribution of current blockade event amplitudes. Specific signatures of oligo (tail) and nanoparticle (head) blocks are clearly identified. Adapted with permission from [19]

DNA strand in solution generated a dramatic change in the rectification coefficient due to hybridization between the probe DNA and complementary strands, whereas a control of polyT addition did not result in any appreciable change in the current voltage curve. Furthermore, their system showed distinguishable signal with single base DNA mismatch. Addition of a mismatched DNA sequence resulted in a lower rectification coefficient than the one observed with perfect complement. A representation of their results is shown in Fig. 6.

Umehara et al. showed two different strategies to employ functionalized nanopipettes as a biosensor [37]. First, they monitored the modulation of the rectification properties of the glass nanopipette upon the electrostatic binding of charged polymers. The second strategy relied on the change in the current amplitude upon binding of target proteins to their corresponding antibodies immobilized at the nanopipette tip. Nanopipettes were first modified with a cationic polyelectrolyte, poly-l-lysine, that electrostatically binds the negatively charged nanopipette walls. The amino groups

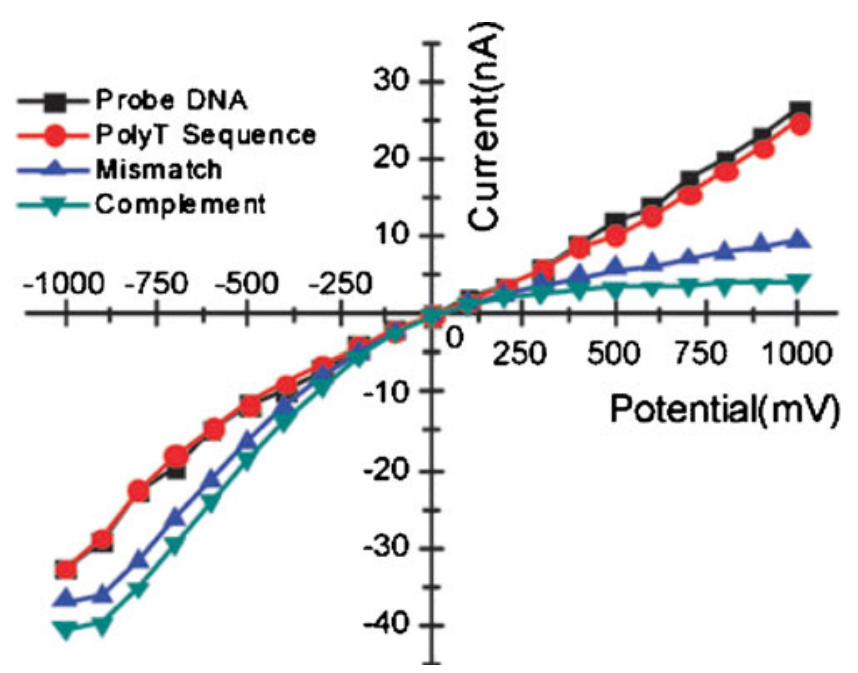

Fig. 6 Adapted results of ref [26] in which the nanopipettes was used as a DNA hybridization sensor. Reproduced by permission of The Royal Society of Chemistry 
allow for the covalent binding of the antibodies through the EDC/NHS cross-linking chemistry. Electrical measurements were performed into a buffered $0.1 \mathrm{M} \mathrm{KCl}$ solution with a sinusoidal applied voltage. They observed that the addition of a small protein (interleukine-10, IL-10) in solution affected the current amplitude but not the rectification polarity (Fig. 7). Unlike highly charged polymers, proteins would mainly have an effect on the current because of their physical size (typical Stokes radius ranges several nanometers) when bound to the functionalized surface and partially blocking the current path.

The goal of their work was to prove that biomolecular interactions can be detected in a label-free manner through nanopipette technology. However, improvement in the technology can be made, for example, by limiting the functionalized surface area to prevent the capture of targets on the sidewalls away from the sensing region. Another option is to take advantage of the accumulation of molecules in and around the tip region under constant voltage [18].

a


Fig. 7 Current amplitude changes upon binding of IL-10 and VEGF to anti-IL-10 and anti-VEGF functionalized nanopipettes. Adapted with permission from [34]
To fully realize the potential of nanopipette as an analytical biosensor, the nanopipette response to analyte concentration should be systematically investigated. Ding et al. proposed an aptamer-encoded nanopipette to detect immunoglobulin $\mathrm{E}$ and a ricin, a $64 \mathrm{kDa}$ glycoprotein toxin, and explored the effect of analyte concentration in this system. For the immobilization of aptamer on the nanopore, they followed a method that was successfully established to immobilize proteins on silicon nanowires [38]. The glass surface in the pore was silanized with aldehyde methoxysilane, followed by attaching amino-terminated DNA or RNA aptamers to the aldehyde-terminated glass surface in sodium cyanoborohydride. The electrical measurements were performed in a PBS solution, and the ionic current at the nanopore was monitored as a function of time under a constant applied voltage.

The authors first identified a series of stepwise blockades on measured current in a nanopipette functionalized with anti-IgE aptamer in presence of $\operatorname{IgE}$ molecules (Fig. 8). Similar blockades were not observed with unfunctionalized nanopipettes or in nanopipettes modified with different aptamers indicating that non-specific adsorption was not observed and the signal measured arises strictly from affinity based bindings. Similar results were obtained with nanopipettes modified with anti-ricin A-chain RNA aptamer in presence of the ricin A-chain.

Interestingly, the authors were the first to address the question about the limit of detection and the linear range of the nanopipette assay. They tried to correlate the analyte concentration with the time interval between the first and second blockade events detected, $\Delta t_{12}=t_{1}-t_{2}$. Assuming that the detection time from each block $t_{n}$ follows the exponential distribution,

$n=n_{0}\left(1-e^{-\frac{t_{n}}{\tau}}\right)$

the constant $\tau$ is inversely proportional to the target concentration by a factor $k_{\text {on }}$ that is the associate rate constant. However, in solutions with low concentration of the detected molecule, the detection time $\Delta t_{12}$ will be

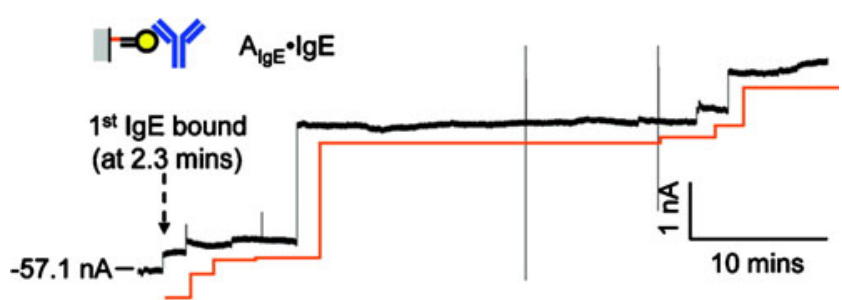

Fig. 8 Stepwise blockade of measured currents upon the individual binding event of immunoglobin $\mathrm{E}$ ( $\mathrm{IgE}$ ) in assay solution to the antiIgE aptamer immobilized on the nanopipette surface. Adapted with permission from [33] 
prolonged because, statistically, fewer events will take place and thus detected in the same duration. The authors showed that the detection time dropped by $130 \mathrm{~min}$ as the concentration of the analyte increased from $500 \mathrm{fM}$ to $5 \mathrm{nM}$.

The large number of candidate materials for the fabrication of nanopipette makes it suitable for many different biosensing applications with the proper surface treatment and chemistry. Here we summarize the recent biosensing studies using the nanopipette platform (Table 1).

\section{Nanopipettes and electrophysiology}

Nanopipettes and micromanipulators can be integrated for single cell imaging and nanoinjection. We will not discuss all the different applications of the scanned nanopipette, as this topic has been recently reviewed by Klenerman and coworkers [9]. However, some interesting features can lead to a unique biosensor platform capable of a sensitive analysis ex vivo down to the single cell level. Hansma and colleagues [39] first demonstrated the ability of glass nanopipette to image the topography of non-conducting surfaces immersed into electrolyte solutions. A variation in current is observed when the nanopipette tip approaches the surface of the sample. Therefore, the measured ionic current can be used as input to a feedback control loop keeping the distance between the tip and the sample constant during scanning [40]. However, small electrolytic changes in electrodes, e.g., accumulation of charged molecules inside the pipette, will also affect the measured current. To address this issue, a distance-modulated control mechanism has been developed for reliable non-contact imaging over the surface of a live cell [41].

Another area of interest is the use of nanopipettes for single cell nanoinjection. There are several advantages over conventional microinjection techniques, including:

1. high cell survival rate

2. precise control of the amount of material delivered

3. possibility of a voltage or pressure driven injection

4. applicability of the platform for the delivery of multiple (bio)molecules
Work from many research groups highlights the advances in the use of nanopipettes for injection. Bruckbauer et al. delivered individual fluorescently labeled probe molecules to the plasma membrane through a nanopipette. Single molecule fluorescence tracking was used to validate the method. They studied the diffusion of individual membrane glycoproteins labeled with a fluorescent dye in different surface domains of boar spermatozoa [42]. Piper et al. demonstrated the local control of sodium-sensitive flagellar motor in single Escherichia coli cells by dosing sodium via a nanopipette [43]. Laforge et al. filled a nanopipette with a waterimmiscible organic solvent and immersed it in an aqueous solution [11]. The electrochemical attosyringe takes advantage of the phenomenon that the application of voltage across the liquid/liquid interface changes the surface tension [44]. The resulting force is sufficiently strong to induce the flow of liquid into/out of the pipette. They have successfully used this effect to deliver femtoliters of aqueous solution into mammalian cells in culture. Cell integrity after injection was confirmed by trypan blue-exclusion. Similar experiments were performed with carbon nanopipettes [45]. Vitol et al. introduced a SERSactive carbon nanopipette for intracellular analysis [46]. SERS functionality is added by incorporating gold nanoparticles on the outer surface pipette tip. The technique allows the accurate tracking of the tip location within the cell. SERS spectra obtained with the nanopipette from within the nucleus are clearly different from those obtained within the cytoplasm and contain typical features associated with DNA.

\section{Conclusions and outlook}

Recently, many researchers have focused on applications of nanopipettes for single cell penetration to study intracellular compartments, and there is a growing interest in the exploration of functional phenomena directly inside a single (pathogenic) cell. Recent advances combine the sensitivity and the selectivity of nanopipettes as a biosensor with the

Table 1 Summary of recent nanopipette biosensing applications

\begin{tabular}{lllll}
\hline Target & Waveform & Limit of Detection & Detection Time & References \\
\hline Small proteins (IL-10, VEGF) & Sine wave & $4 \mu \mathrm{g} / \mathrm{ml}$ & Seconds & Not mentioned \\
DNA & Linear sweep & $\sim \mu \mathrm{M}$ & $>130 \mathrm{~min}$ at 500 fM & {$[26]$} \\
IgE, Ricin & Constant voltage & $500 \mathrm{fM}$ (IgE) & [33] & Not mentioned \\
DNA labeled with Au nanoparticle & Constant voltage & Not investigated & & \\
\hline
\end{tabular}


ease and the precision of manipulation of such sensor into well-defined region of a cell surface or compartment. Although nanopipettes are recent inventions, they are a promising tool for combining single cell analysis and cell manipulation. We believe that the nanopipette will become an essential tool for electrophysiological and medical research in the near future.

Acknowledgments This work was supported in part by grants from the National Aeronautics and Space Administration Cooperative Agreements NCC9-165 and NNX08BA47A, National Institutes of Health [P01-HG000205], the National Science Foundation [DBI 0830141].

Open Access This article is distributed under the terms of the Creative Commons Attribution Noncommercial License which permits any noncommercial use, distribution, and reproduction in any medium, provided the original author(s) and source are credited.

\section{References}

1. Monk DJ, Walt DR (2004) Optical fiber-based biosensors. Anal Bioanal Chem 379:931-945

2. Ghica ME, Pauliukaite R, Fatibello-Filho O, Brett CMA (2009) Application of functionalised carbon nanotubes immobilised into chitosan films in amperometric enzyme biosensors. Sens actuators B Chem 142:308-315

3. Hahm JI, Lieber CM (2003) Direct ultrasensitive electrical detection of DNA and DNA sequence variations using nanowire nanosensors. Nano Lett 4:51-54

4. Rosi NL, Mirkin CA (2005) Nanostructures in biodiagnostics. Chem Rev 105:1547-1562

5. Sutter PW, Sutter EA (2007) Dispensing and surface-induced crystallization of zeptolitre liquid metal-alloy drops. Nat Mater 6:363-366

6. Stulík K, Amatore C, Holub K, Marecek V, Kutner W (2000) Microelectrodes. Definitions, characterization, and applications (Technical report). Pure Appl Chem 72:1483-1492

7. Cunningham A (2000) Introduction to Bioanalytical Sensors. Wiley, NY, p 29.

8. Yin L (2009) Applications of nanopipettes in bionanotechnology. Biochem Soc Trans 37:702-706

9. Klenerman D, Korchev Y (2006) Potential biomedical applications of the scanned nanopipette. Nanomedicine (Lond) 1:107-114

10. Calander N (2009) Analyte concentration at the tip of a nanopipette. Anal Chem 81:8347-8353

11. Laforge FO, Carpino J, Rotenberg SA, Mirkin MV (2007) Electrochemical attosyringe. Proc Natl Acad Sci 104:11895-11900

12. Gorelik J, Shevchuk A, Ramalho M, Elliott M, Lei C, Higgins CF, Lab MJ, Klenerman D, Krauzewicz N, Korchev Y (2002) Scanning surface confocal microscopy for simultaneous topographical and fluorescence imaging: application to single virus-like particle entry into a cell. Proc Natl Acad Sci USA 99:16018-16023

13. Comstock DJ, Elam JW, Pellin MJ, Hersam MC (2010) Integrated ultramicroelectrode-nanopipet probe for concurrent scanning electrochemical microscopy and scanning ion conductance microscopy. Anal Chem 82:1270-1276

14. Clarke RW, Piper JD, Ying L, Klenerman D (2007) Surface conductivity of biological macromolecules measured by nanopipette dielectrophoresis. Phys Rev Lett 98:198102
15. Piper JD, Clarke RW, Korchev YE, Ying L, Klenerman D (2006) A renewable nanosensor based on a glass nanopipette. J Am Chem Soc 128:16462-16463

16. Iwata F, Nagami S, Sumiya Y, Sasaki A (2007) Nanometre-scale deposition of colloidal $\mathrm{Au}$ particles using electrophoresis in a nanopipette probe. Nanotechnology 18:105301

17. Gates BD, Xu Q, Stewart M, Ryan D, Willson CG, Whitesides GM (2005) New approaches to nanofabrication: molding, printing, and other techniques. Chem Rev 105:1171-1196

18. Clarke RW, White SS, Zhou D, Ying L, Klenerman D (2005) Trapping of proteins under physiological conditions in a nanopipette. Angew Chem Int Ed Engl 44:3747-3750

19. Munoz JL, Coles JA (1987) Quartz micropipettes for intracellular voltage microelectrodes and ion-selective microelectrodes. J Neurosci Meth 22:57-64

20. Rae JL, Levis RA (1992) A method for exceptionally low noise single channel recordings. Pflügers Arch Eur J Physiol 420:618-620

21. Levis RA, Rae JL (1993) The use of quartz patch pipettes for low noise single channel recording. Biophys J 65:1666-1677

22. Zuazaga C, Steinacker A (1990) Patch-clamp recording of ion channels: interfering effects of patch pipette glass. News Physiol Sci 65:1666-1677

23. Karhanek M, Kemp JT, Pourmand N, Davis RW, Webb CD (2005) Single DNA molecule detection using nanopipettes and nanoparticles. Nano Lett 5:403-407

24. Gao C, Ding S, Tan Q, Gu LQ (2008) Method of creating a nanopore-terminated probe for single-molecule enantiomer discrimination. Anal Chem 81:80-86

25. Kim BM, Murray T, Bau HH (2005) The fabrication of integrated carbon pipes with sub-micron diameters. Nanotechnology 16:1317-1320

26. Freedman JR, Mattia D, Korneva G, Gogotsi Y, Friedman G, Fontecchio AK (2007) Magnetically assembled carbon nanotube tipped pipettes. Appl Phys Lett 90:103108

27. Wei C, Bard AJ, Feldberg SW (1997) Current rectification at quartz nanopipet electrodes. Anal Chem 69:4627-4633

28. Umehara S, Pourmand N, Webb CD, Davis RW, Yasuda K, Karhanek M (2006) Current rectification with poly-l-lysine-coated quartz nanopipettes. Nano Lett 6:2486-2492

29. Bard AJ, Faulkner LR (eds) (1980) Electrochemical methods: fundamentals and applications. Wiley, New York

30. Fu Y, Tokuhisa H, Baker LA (2009) Nanopore DNA sensors based on dendrimer-modified nanopipettes. Chem Commun (Camb) 32:4877-4879

31. Sexton LT, Horne LP, Sherrill SA, Bishop GW, Baker LA, Martin CR (2007) Resistive-pulse studies of proteins and protein/ antibody complexes using a conical nanotube sensor. J Am Chem Soc 129:13144-13152

32. Sakmann ENB (ed) (1995) Single-channel recording. New York

33. Lavallée OSM, Hébert N (ed) (1969) Glass microlectrodes. New York

34. Ding S, Gao C, Gu LQ (2009) Capturing single molecules of immunoglobulin and ricin with an aptamer-encoded glass nanopore. Anal Chem 81:6649-6655

35. Smeets RMM, Keyser UF, Wu MY, Dekker NH, Dekker C (2006) Nanobubbles in solid-state nanopores. Phys Rev Lett 97:088101

36. Tabard-Cossa V, Trivedi D, Wiggin M, Jetha NN, Marziali A (2007) Noise analysis and reduction in solid-state nanopores. Nanotechnology 18:305505

37. Umehara S, Karhanek M, Davis RW, Pourmand N (2009) Labelfree biosensing with functionalized nanopipette probes. Proc Natl Acad Sci 106:4611-4616

38. Patolsky F, Zheng G, Hayden O, Lakadamyali M, Zhuang X, Lieber CM (2004) Electrical detection of single viruses. Proc Natl Acad Sci USA 101:14017-14022 
39. Hansma P, Drake B, Marti O, Gould S, Prater C (1989) The scanning ion-conductance microscope. Science 243:641-643

40. Bocker M, Anczykowski B, Wegener J, Schaffer TE (2007) Scanning ion conductance microscopy with distance-modulated shear force control. Nanotechnology 18:145505

41. Liming Ying AB, Zhou D, Gorelik J, Shevchuk A, Lab M, Korchev Y, Klenerman D (2005) The scanned nanopipette: a new tool for high resolution bioimaging and controlled deposition of biomolecules. PCCP 7:2859-2866

42. Bruckbauer A, James P, Zhou D, Yoon JW, Excell D, Korchev Y, Jones R, Klenerman D (2007) Nanopipette delivery of individual molecules to cellular compartments for single-molecule fluorescence tracking. Biophys J 93:3120-3131
43. Piper JD, Li C, Lo CJ, Berry R, Korchev Y, Ying L, Klenerman D (2008) Characterization and application of controllable local chemical changes produced by reagent delivery from a nanopipet. J Am Chem Soc 130:10386-10393

44. Girault H, Schiffrin DJ (eds) (1989) Electroanalytical chemistry. Marcel Dekker, New York

45. Schrlau MG, Falls EM, Ziober BL, Bau HH (2008) Carbon nanopipettes for cell probes and intracellular injection. Nanotechnology 19:015101

46. Vitol EA, Orynbayeva Z, Bouchard MJ, Azizkhan-Clifford J, Friedman G, Gogotsi Y (2009) In situ intracellular spectroscopy with surface enhanced raman spectroscopy (SERS)-enabled nanopipettes. ACS Nano 3:3529-3536 